\title{
Successful management of spinal cord ischemia in a pediatric patient with fibrocartilaginous embolism: illustrative case
}

\author{
*Augustinas Fedaravičius, MD, ${ }^{1,2}$ Yael Feinstein, MD, ${ }^{3}$ Isaac Lazar, MD, ${ }^{3}$ Micky Gidon, MD, ${ }^{1}$ llan Shelef, MD, ${ }^{4}$ Elad Avraham, MD, \\ Arimantas Tamašauskas, $\mathrm{MD}, \mathrm{PhD}^{2}$ and Israel Melamed, $\mathrm{MD}^{1}$ \\ ${ }^{1}$ Department of Neurosurgery, ${ }^{3}$ Pediatric Intensive Care Unit, and ${ }^{4}$ Department of Radiology, Soroka Medical Center, Be'er Sheva, Israel; and ${ }^{2}$ Department of Neurosurgery, \\ Hospital of Lithuanian University of Health Sciences, Kaunas, Lithuania
}

BACKGROUND Fibrocartilaginous embolism (FCE) is a rare cause of ischemic myelopathy that occurs when the material of the nucleus pulposus migrates into vessels supplying the spinal cord. The authors presented a case of pediatric FCE that was successfully managed by adapting evidencebased recommendations used for spinal cord neuroprotection in aortic surgery.

OBSERVATIONS A 7-year-old boy presented to the emergency department with acute quadriplegia and hemodynamic instability that quickly progressed to cardiac arrest. After stabilization, the patient regained consciousness but remained in a locked-in state with no spontaneous breathing. The patient presented a diagnostic challenge. Traumatic, inflammatory, infectious, and ischemic etiologies were considered. Eventually, the clinical and radiological findings led to the presumed diagnosis of FCE. Treatment with continuous cerebrospinal fluid drainage (CSFD), pulse steroids, and mean arterial pressure augmentation was applied, with subsequent considerable and consistent neurological improvement.

LESSONS The authors proposed consideration of the adaptation of spinal cord neuroprotection principles used routinely in aortic surgery for the management of traumatic spinal cord ischemia (FCE-related in particular), namely, permissive arterial hypertension and CSFD. This is hypothesized to allow for the maintenance of sufficient spinal cord perfusion until adequate physiological blood perfusion is reestablished (remodeling of the collateral arterial network and/or clearing/absorption of the emboli).

https://thejns.org/doi/abs/10.3171/CASE21380

KEYWORDS spinal cord ischemia; fibrocartilaginous embolism; spinal cord perfusion pressure

Spinal cord ischemia (SCI), or spinal infarct, is a rare but debilitating disorder caused by a wide variety of etiologies. Over the last decades, significant research has been conducted on the pathophysiology and prevention of SCl; however, current clinical management of this pathology remains rooted in methods derived from cerebral stroke and spinal trauma. ${ }^{1-7}$ Nonetheless, principles of neuroprotection based on increasing spinal cord blood flow (SCBF) have been successfully implemented in thoracoabdominal aortic surgery. ${ }^{8-15}$ We were prompted to apply these principles to treat a pediatric patient with cervical $\mathrm{SCl}$ due to fibrocartilaginous embolism (FCE). FCE, first reported in $1961,{ }^{16}$ is a rare but potentially underdiagnosed cause of ischemic myelopathy. ${ }^{17,18}$
It occurs when material of the nucleus pulposus migrates into vessels supplying the spinal cord. ${ }^{17,19-21}$

\section{Illustrative Case}

A 7-year-old boy was admitted to the ER with reports of back pain, progressive weakness, and vomiting that started abruptly after playful wrestling with his brother. In the emergency department, the patient presented with signs of high spinal cord injury causing neurogenic shock, cyanosis, bradycardia, hypothermia, and hypotension nonresponsive to isotonic crystalloid fluid boluses. He deteriorated rapidly into cardiac arrest. Successful advanced cardiopulmonary

\footnotetext{
ABBREVIATIONS CSF = cerebrospinal fluid; CSFD = CSF drainage; DWI = diffusion-weighted imaging; FCE = fibrocartilaginous embolism; MAP = mean arterial pressure; $\mathrm{MRI}=$ magnetic resonance imaging; $\mathrm{SCBF}=$ spinal cord blood flow; $\mathrm{SCl}$ = spinal cord ischemia; $\mathrm{SCPP}=$ spinal cord perfusion pressure . INCLUDE WHEN CITING Published September 13, 2021; DOI: 10.3171/CASE21380.

SUBMITTED June 26, 2021. ACCEPTED July 29, 2021.

* A.F. and Y.F. contributed equally to this work.

(C) 2021 The authors, CC BY-NC-ND 4.0 (http://creativecommons.org/licenses/by-nc-nd/4.0/).
} 

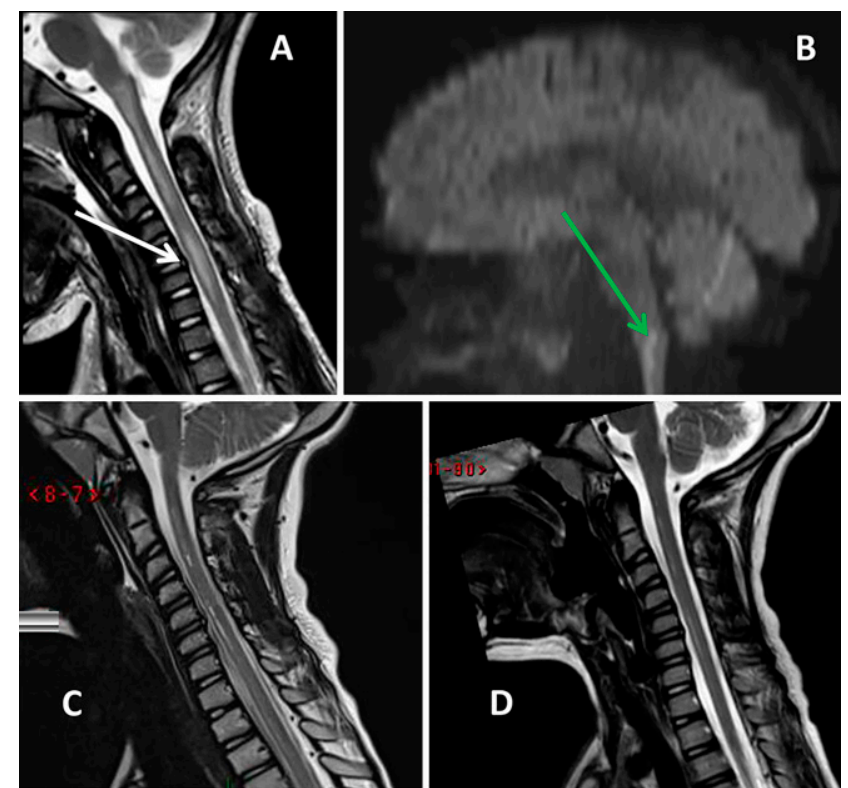

FIG. 1. A and B: MRI sequences obtained after initial presentation Hyperintensity in T2-weighted sequences (A) as well as diffusion restriction ( $\mathbf{B}$, green arrow) can be seen. Those findings suggest an ischemic etiology. The white arrow $(\mathbf{A})$ indicates an annular tear in the C6-C7 intervertebral disc. C and D: Follow-up MRI study (C, 4 months after event; D, 11 months after event).

Postischemic changes in the medulla oblongata and the spinal cord are visible.

resuscitation restored spontaneous circulation. The patient was transferred to the pediatric intensive care unit, where he was ventilated and supported with continuous infusion of noradrenaline.

Brain, cervical spine, and thoracic computed tomography scans were unremarkable other than showing small bilateral consolidations in the lungs. With resolving signs of shock, continuous infusion of milrinone was commenced as noradrenaline was tapered off.
The child regained consciousness after sedation was ceased. He was fully oriented and communicated with his parents using eye movements (locked-in syndrome). On physical examination, flaccid quadriplegia and areflexia were noted, with preserved normal pinprick and deep sensations (confirmed by blinking). He had no spontaneous breathing movements.

The patient presented a diagnostic challenge. Acute neurogenic shock with flaccid paralysis and intact sensation was believed to be secondary to traumatic, inflammatory, infectious, or ischemic etiologies. Cerebrospinal fluid (CSF) analysis revealed no pleocytosis, normal glucose concentration (90 mg/dL), and low protein (11 mg/dL). Oligoclonal bands, antimyelin basic protein, and paraneoplastic panel results were all negative. Those results decreased the likelihood of an inflammatory or infectious etiology. Neurosurgeons were consulted, and total-axis magnetic resonance imaging (MRI) was performed on the third day of admission. A hyperintensity in T2-weighted sequences and restriction in diffusion-weighted imaging (DWI) sequences were demonstrated, located most prominently at the level of $\mathrm{C} 6$ and extending from the medulla oblongata proximally to the level of T1 caudally. Furthermore, adjacent to the most pronounced signs of pathological MRI signal at the C6 level, a C6-C7 annular tear without spinal cord compression was demonstrated (Fig. 1). No spinal ligamentous traumatic injuries were demonstrated.

Corticosteroids were commenced (dexamethasone $0.2 \mathrm{mg} / \mathrm{kg}$ per day) to reduce any vasogenic edema.

Because improvement was minimal, after another revision of the data, which suggested an ischemic insult with distribution of anterior spinal cord syndrome, the diagnosis of FCE was proposed. On the evening of the fourth day of admission, a continuous CSF drainage (CSFD) catheter was inserted. CSF pressure of 5 to $10 \mathrm{~mm} \mathrm{Hg}$ was maintained throughout the duration of therapy.

On the next day, spontaneous breathing as well as considerable improvement in gross motor strength and reflexes were noted. CSFD was continued for 2 days. No CFSD-related complications were observed. The timeline of treatment and neurological status is presented in Fig. 2.

Because an inflammatory process could not be ruled out completely, after a multidisciplinary team discussion, a course of pulse

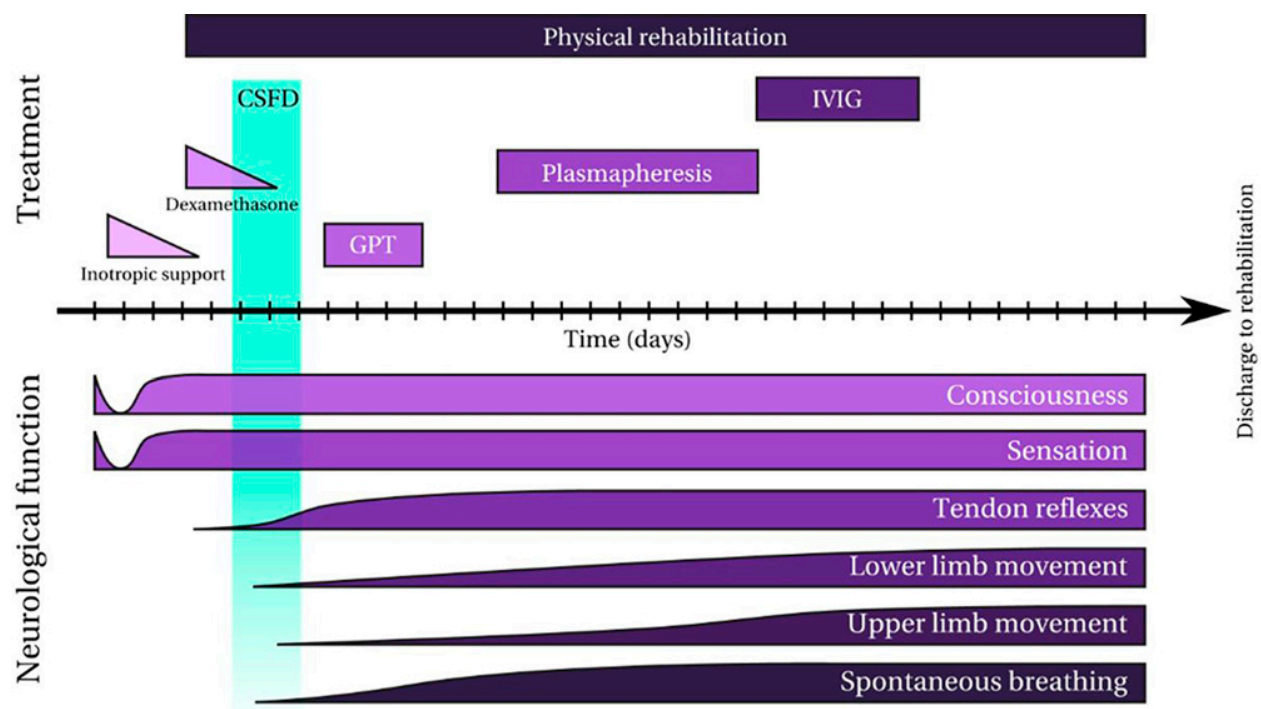

FIG. 2. The timeline of the case from arrival to the emergency department to discharge from pediatric intensive care unit. GPT = glucocorticoid pulse therapy; IVIG = intravenous immunoglobulin. 
therapy with methylprednisolone (10 mg/kg for 5 days) was commenced followed by a course of plasmapheresis (over 5 alternating days) and intravenous immunoglobulin. However, the greatest improvement was achieved during the CSFD session.

Because of ongoing ventilatory requirements, a tracheostomy was performed. The patient continued to improve neurologically and was gradually weaned off ventilatory support. Eventually, he was discharged to a rehabilitation center.

At the 6-month follow-up visit, the patient had normal cranial nerve function; muscle strength was $4+$ in the right extremities and 4- in the left; normal muscle tone with no spasticity in the arms and slight spasticity in both legs was observed. Deep pain and temperature sensations were preserved.

Follow-up MRI showed postischemic changes in the medulla and spinal cord (Fig. 1).

\section{Discussion}

The rarity of FCE along with its complex pathophysiology raises a significant diagnostic challenge, as illustrated in this case. Moreover, once diagnosis is made, the paucity of proposed, let alone evidence-based, recommendations complicates treatment decisions.

Spinal cord infarction is a rare pathology in itself, representing only up to $1 \%$ of all strokes. ${ }^{22}$ Out of these, the occurrence of FCE is reported to be $1.8-5.5 \% .{ }^{22,23}$ However, some clinicians believe that these numbers are an underestimation. ${ }^{17,18}$

FCE demonstrates a bimodal pattern of presentation, with peak incidence in adolescence and middle age, most likely because of the pattern of vascularization of the intervertebral disc. ${ }^{17,19}$ It shows a slight female predominance. ${ }^{17,19}$

The mechanism of FCE includes the migration of nucleus pulposus material into vessels supplying the spinal cord, as illustrated in Fig. 3 .

The typical presentation includes transient neck or back pain followed by neurological deficits that depend on the vascular territory of the occluded vessel, ${ }^{17}$ most commonly the anterior spinal artery, which usually results in bilateral motor deficit with possible asymmetrical presentation and spinothalamic sensory deficit. ${ }^{22,24}$

Definitive diagnosis of FCE is only possible through histological examination and is usually made postmortem. ${ }^{19,20,23,25}$ There is one reported case of diagnosis obtained via spinal cord biopsy, ${ }^{26}$ which is generally not recommended because of the high risk of additional neurological damage. ${ }^{25,27}$ Therefore, the diagnosis of FCE is generally made by exclusion. ${ }^{17,20,25}$

CSF analysis is recommended in all cases of suspected FCE. ${ }^{17,22}$ The results may be normal ${ }^{18}$ but typically show elevated protein levels. ${ }^{17}$ CSF analysis in FCE differs from inflammatory etiologies (autoimmune/infectious) in that no pleocytosis, oligoclonal bands, or elevated IgG index are present. ${ }^{17}$ Another diagnostic clue that aids in the differentiation of FCE from inflammatory conditions is derived from the faster development of symptoms in FCE (minutes to days as opposed to weeks) that precedes radiological changes. ${ }^{17,19,23}$

Typically, MRI shows hyperintense signal conversion in T2-weighted sequences, with spinal cord swelling and hyperintense signal changes (possibly due to hemorrhagic transformation) in T1-weighted sequences. Scattered T1 gadolinium enhancement is also possible; however, the appearance is different from the pattern occurring in lesions of inflammatory origin. ${ }^{22}$ In the acute stage, restricted diffusion on DWI is an especially useful sign because it shows cytotoxic changes in the spinal cord and typically does not appear in cases of transverse myelitis. ${ }^{22,28,29}$ In FCE, these signs are often found adjacent to degenerative

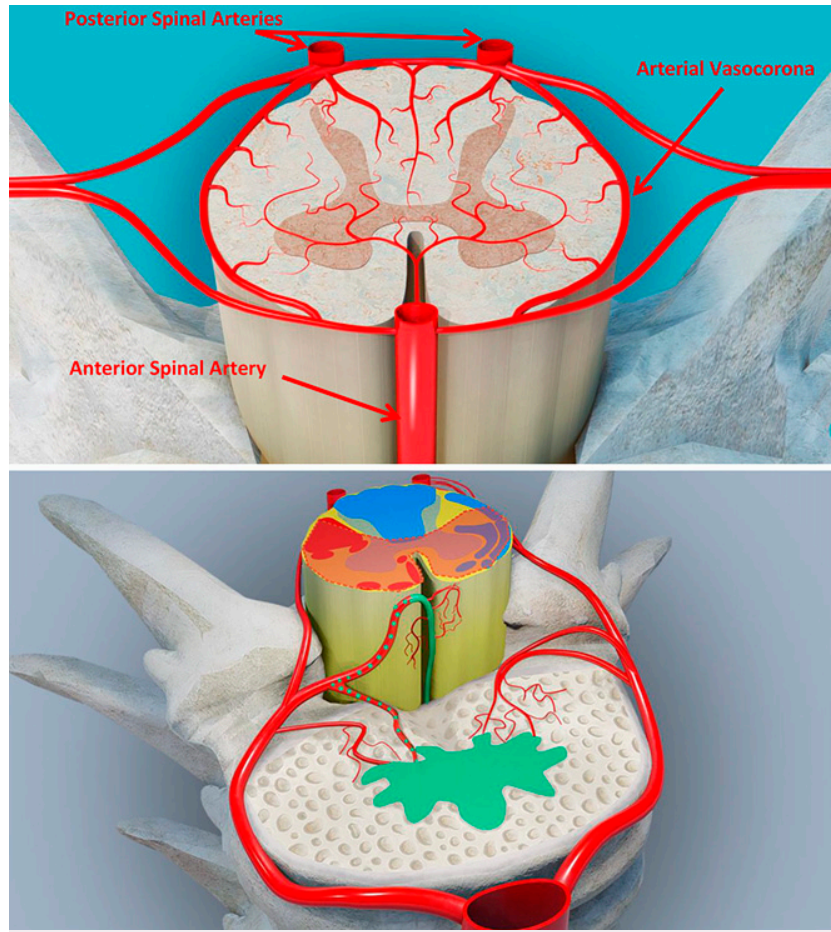

FIG. 3. Upper: Arterial supply of the cervical spinal cord. The anterior spinal artery supplies the anterior two-thirds of the spinal cord and low medulla. Paired posterior spinal arteries supply the posterior third of the spinal cord. Arterial vasocorona provides delicate anastomoses on the pial surface that send branches supplying the peripheral portions of the lateral funiculi. Lower: Illustration of the arterial mechanism of FCE. During activities that involve elevation of axial load (with subsequent elevated intradiscal pressure), material from the intervertebral disk (depicted in green) travels in a retrograde fashion to the radicular arteries, then anterograde to the arterial system of the spinal cord, thus leading to anterior spinal artery syndrome. The resulting ischemic territory is shaded in red.

changes or minor traumatic findings of the intervertebral discs (as described in the current case). ${ }^{17}$

The absence of high-energy injury and radiological evidence of major trauma (bony and/or ligamentous), along with significant and incompatible neurological impairment, helps with the exclusion of a direct traumatic injury to the spinal cord.

The algorithm for the clinical diagnosis of FCE has been described elsewhere (AbdelRazek et al. ${ }^{17}$ ) and can be outlined as follows: (1) Establish the diagnosis of myelopathy and the affected level. (2) Exclude traumatic and compressive etiologies. (3) Exclude inflammatory etiologies. (4) Establish the diagnosis of SCl. (5) Establish the high likelihood of FCE (related minor spinal trauma or episode of increased intradiscal pressure and/or Valsalva maneuver, presence of degenerative disc disease, especially Schmorl's nodes, at or near the level of myelopathy, and no more than one cardiovascular risk factor).

Currently, no specific treatment for FCE has been proposed. Therefore, we were prompted to seek possible management options in the broader context of SCl.

The most common etiology of $\mathrm{SCl}$ is pathology of the aorta (atherosclerosis, dissection, and procedure-related causes). ${ }^{8,22}$ Accordingly, 
most of the significant studies regarding the prevention and treatment of $\mathrm{SCl}$ have been done in this context.

Emerging treatment options for $\mathrm{SCl}$ consist of the following strategies: (1) hypothermia, (2) systemic hypertension, (3) CSFD via lumbar drain, (4) ischemic preconditioning, (5) hyperbaric oxygen therapy, and (6) pharmacological neuroprotection. ${ }^{8}$

We focus on the use of permissive systemic hypertension and CSFD with the intention to increase the spinal cord perfusion pressure (SCPP) and SCBF. SCPP is defined as

$$
\mathrm{SCPP}=\mathrm{MAP}-\max \left\{\mathrm{P}_{\mathrm{CSF}}, \mathrm{P}_{\mathrm{RA}}\right\}
$$

where MAP is the mean arterial pressure, $P_{C S F}$ is the CSF pressure in the spinal canal, $P_{R A}$ is the pressure in the right atrium, and $\max \{a, b\}$ is a function that returns the greater of the two inputs. ${ }^{13}$ SCBF is a parameter with a much more complex nonlinear dependence on MAP, $\mathrm{CO}_{2}$ concentration, and autoregulation properties. ${ }^{5,6}$

Results of animal studies have demonstrated that systemic hypotension significantly potentiates neurological dysfunction after temporary aortic occlusion. ${ }^{1}$ Increasing systemic blood pressure during $\mathrm{SCl}$ can reduce ischemic damage and postoperative neurological adverse events. ${ }^{4}$ Permissive arterial hypertension sustained for at least 24 to 48 hours after aortic surgery provides increased spinal cord perfusion during the necessary time for the aforementioned anatomical adaptations to take place. ${ }^{13}$ Obviously, maintaining sufficient arterial blood pressure is especially important in patients who develop neurogenic shock. A similar approach to traumatic spinal cord injury with aggressive resuscitation and blood pressure management in addition to surgery has been described in one case series with good clinical results. ${ }^{30}$

CSFD as a means of preventing $\mathrm{SCl}$ has been extensively analyzed in animal models, prospective clinical studies, and systematic reviews. ${ }^{13}$ The 2010 American Heart Association strongly recommends (Class I, B level evidence) CSFD for the prevention of SCI for aorta surgery in high-risk patients. ${ }^{15} \mathrm{~A}$ meta-analysis performed by Khan et al. ${ }^{14}$ found that CSFD reduces SCl by almost half. The usual target CSF pressure is $10 \mathrm{~mm} \mathrm{Hg}$, with the drainage rate no more than $25 \mathrm{~mL} / \mathrm{h}^{13,14}$

In their pig model-based study, Martirosyan et al. demonstrated that MAP elevation or CSFD alone provided only short-term improvement of SCBF. However, when both interventions were performed concurrently, SCBF and SCPP were improved significantly and sustainably. ${ }^{3}$

Another pig model-based study demonstrated that as few as 24 hours are required for significant collateral artery formation/remodeling after segmental spinal artery occlusion with resultant significant improvement in SCBF. ${ }^{7}$ Collateral blood supply formation of the human spinal cord is illustrated in Fig. 3.

\section{Observations}

Based on the literature review and clinical case presented, we offer the following recommendations for the treatment of SCl: (1) Hemodynamic stabilization/MAP augmentation: intravenous fluids, vasopressors, inotropes. maintenance of MAP at 85 to $100 \mathrm{~mm} \mathrm{Hg}$. (2) Insertion of a lumbar continuous CSF drainage system for 48 to 72 hours with target CSF pressure of $10 \mathrm{~mm} \mathrm{Hg}$ and a drainage rate no more than $20 \mathrm{~mL} / \mathrm{h}$. (3) Glucocorticoid pulse therapy for 5 to 7 days.

\section{Lessons}

We propose the adaptation of spinal cord neuroprotection principles used routinely in aortic surgery for the management of traumatic SCl (FCE-related, in particular), namely, permissive arterial hypertension and CSFD, to allow for adequate SCPP until physiological blood perfusion is reestablished.

\section{Acknowledgments}

We would like to thank artist Sergey Khvostenko for the threedimensional rendered illustration of spinal vascular anatomy and the mechanism of FCE.

\section{References}

1. Taira $Y$, Marsala M. Effect of proximal arterial perfusion pressure on function, spinal cord blood flow, and histopathologic changes after increasing intervals of aortic occlusion in the rat. Stroke. 1996;27(10):1850-1858.

2. Martirosyan NL, Feuerstein JS, Theodore N, Cavalcanti DD, Spetzler RF, Preul MC. Blood supply and vascular reactivity of the spinal cord under normal and pathological conditions. J Neurosurg Spine. 2011;15(3):238-251.

3. Martirosyan NL, Kalani MYS, Bichard WD, et al. Cerebrospinal fluid drainage and induced hypertension improve spinal cord perfusion after acute spinal cord injury in pigs. Neurosurgery. 2015;76(4): 461-469.

4. Izumi S, Okada K, Hasegawa T, et al. Augmentation of systemic blood pressure during spinal cord ischemia to prevent postoperative paraplegia after aortic surgery in a rabbit model. $J$ Thorac Cardiovasc Surg. 2010;139(5):1261-1268.

5. Marcus ML, Heistad DD, Ehrhardt JC, Abboud FM. Regulation of total and regional spinal cord blood flow. Circ Res. 1977;41(1): 128-134.

6. Gallagher MJ, Hogg FRA, Zoumprouli A, Papadopoulos MC, Saadoun S. Spinal cord blood flow in patients with acute spinal cord injuries. J Neurotrauma. 2019;36(6):919-929.

7. Etz CD, Kari FA, Mueller CS, Brenner RM, Lin H-M, Griepp RB. The collateral network concept: remodeling of the arterial collateral network after experimental segmental artery sacrifice. J Thorac Cardiovasc Surg. 2011;141(4):1029-1036.

8. Nardone R, Pikija S, Mutzenbach JS, et al. Current and emerging treatment options for spinal cord ischemia. Drug Discov Today. 2016;21(10):1632-1641.

9. Pasqualucci A, Al-Sibaie A, Vaidyan KPT, et al. Epidural corticosteroids, lumbar spinal drainage, and selective hemodynamic control for the prevention of spinal cord ischemia in thoracoabdominal endovascular aortic repair: a new clinical protocol. Adv Ther. 2020;37(1):272-287.

10. Evaniew N, Mazlouman SJ, Belley-Côté EP, Jacobs WB, Kwon BK. Interventions to optimize spinal cord perfusion in patients with acute traumatic spinal cord injuries: a systematic review. J Neurotrauma. 2020;37(9):1127-1139.

11. Acher C, Acher CW, Marks E, Wynn M. Intraoperative neuroprotective interventions prevent spinal cord ischemia and injury in thoracic endovascular aortic repair. J Vasc Surg. 2016;63(6):1458-1465.

12. Sutherland BA, Minnerup J, Balami JS, Arba F, Buchan AM, Kleinschnitz $C$. Neuroprotection for ischaemic stroke: translation from the bench to the bedside. Int J Stroke. 2012;7(5):407-418.

13. Augoustides JGT, Stone ME, Drenger B. Novel approaches to spinal cord protection during thoracoabdominal aortic interventions. Curr Opin Anaesthesiol. 2014;27(1):98-105.

14. Khan NR, Smalley Z, Nesvick CL, Lee SL, Michael LM II. The use of lumbar drains in preventing spinal cord injury following thoracoabdominal aortic aneurysm repair: an updated systematic review and meta-analysis. J Neurosurg Spine. 2016;25(3):383-393. 
15. Hiratzka LF, Bakris GL, Beckman JA, et al. 2010 ACCF/AHA/AATS/ ACR/ASA/SCA/SCAI/SIR/STS/SVM Guidelines for the diagnosis and management of patients with thoracic aortic disease. A report of the American College of Cardiology Foundation/American Heart Association Task Force on Practice Guidelines, American Association for Thoracic Surgery, American College of Radiology, American Stroke Association, Society of Cardiovascular Anesthesiologists, Society for Cardiovascular Angiography and Interventions, Society of Interventional Radiology, Society of Thoracic Surgeons, and Society for Vascular Medicine. J Am Coll Cardiol. 2010;55(14): e27-e129.

16. Naiman JL, Donohue WL, Prichard JS. Fatal nucleus pulposus embolism of spinal cord after trauma. Neurology. 1961;11(1):83-87.

17. AbdelRazek MA, Mowla A, Faroog S, Silvestri N, Sawyer R, Wolfe G. Fibrocartilaginous embolism: a comprehensive review of an under-studied cause of spinal cord infarction and proposed diagnostic criteria. J Spinal Cord Med. 2016;39(2):146-154.

18. Ahluwalia R, Hayes L, Chandra T, Maugans TA. Pediatric fibrocartilaginous embolism inducing paralysis. Childs Nerv Syst. 2020; 36(2):441-446.

19. Yamaguchi $H$, Nagase $H$, Nishiyama $M$, et al. Fibrocartilaginous embolism of the spinal cord in children: a case report and review of literature. Pediatr Neurol. 2019;99:3-6.

20. Tosi L, Rigoli G, Beltramello A. Fibrocartilaginous embolism of the spinal cord: a clinical and pathogenetic reconsideration. J Neurol Neurosurg Psychiatry. 1996;60(1):55-60.

21. Quinn JN, Breit H, Dafer RM. Spinal cord infarction due to fibrocartilaginous embolism: a report of 3 cases. J Stroke Cerebrovasc Dis. 2019;28(6):e66-e67.

22. Weidauer S, Nichtweiß M, Hattingen E, Berkefeld J. Spinal cord ischemia: aetiology, clinical syndromes and imaging features. Neuroradiology. 2015;57(3):241-257.

23. Mateen FJ, Monrad PA, Hunderfund ANL, Robertson CE, Sorenson EJ. Clinically suspected fibrocartilaginous embolism: clinical characteristics, treatments, and outcomes. Eur J Neurol. 2011;18(2): 218-225.

24. Novy J, Carruzzo A, Maeder P, Bogousslavsky J. Spinal cord ischemia: clinical and imaging patterns, pathogenesis, and outcomes in 27 patients. Arch Neurol. 2006;63(8):1113-1120.

25. Han JJ, Massagli TL, Jaffe KM. Fibrocartilaginous embolism: an uncommon cause of spinal cord infarction: a case report and review of the literature. Arch Phys Med Rehabil. 2004;85(1):153-157.
26. Mikulis DJ, Ogilvy CS, McKee A, Davis KR, Ojeman RG. Spinal cord infarction and fibrocartilaginous emboli. AJNR Am J Neuroradiol. 1992;13(1):155-160.

27. Davis GA, Klug GL. Acute-onset nontraumatic paraplegia in childhood: fibrocartilaginous embolism or acute myelitis? Childs Nerv Syst. 2000;16(9):551-554.

28. Manara R, Calderone M, Severino MS, et al. Spinal cord infarction due to fibrocartilaginous embolization: the role of diffusion weighted imaging and short-tau inversion recovery sequences. J Child Neurol. 2010;25(8):1024-1028.

29. Thurnher MM, Bammer R. Diffusion-weighted MR imaging (DWI) in spinal cord ischemia. Neuroradiology. 2006;48(11):795-801.

30. Vale FL, Burns J, Jackson AB, Hadley MN. Combined medical and surgical treatment after acute spinal cord injury: results of a prospective pilot study to assess the merits of aggressive medical resuscitation and blood pressure management. J Neurosurg. 1997;87(2):239-246.

\section{Disclosures}

The authors report no conflict of interest concerning the materials or methods used in this study or the findings specified in this paper.

\section{Author Contributions}

Conception and design: Melamed, Fedaravičius, Feinstein, Lazar, Gidon, Shelef. Acquisition of data: Melamed, Fedaravičius, Feinstein, Lazar, Gidon. Analysis and interpretation of data: Melamed, Fedaravičius, Lazar, Gidon. Drafting the article: Melamed, Fedaravičius, Feinstein, Lazar, Shelef, Avraham. Critically revising the article: Melamed, Fedaravičius, Feinstein, Lazar, Gidon, Shelef, Tamašauskas. Reviewed submitted version of manuscript: Melamed, Fedaravičius, Feinstein, Gidon, Shelef, Avraham. Approved the final version of the manuscript on behalf of all authors: Melamed. Administrative/technical/ material support: Melamed, Feinstein. Study supervision: Melamed, Shelef.

\section{Correspondence}

Israel Melamed: Soroka University Medical Center, Be'er Sheva, Israel. melamedi@bgu.ac.il. 